\title{
The management of haematometra secondary to cervical stenosis
}

\author{
Anev Ivan*, Adegbenro Adewale, Awala Alero \\ Department of Obstetrics and Gynaecology, Watford General Hospital, Watford, UK \\ Email: *dranev@doctors.org.uk
}

Received 20 July 2012; revised 23 August 2012; accepted 31 August 2012

\begin{abstract}
This is a case of adolescent cervical stenosis illustrating a possible strong relationship with Depo-Provera use. The case report and its discussion part give an opportunity to debate the way the diagnosis was established, the treatment implemented and the possible underlying cause implied in the pathogenesis. We believe this case would represent an interesting reading material to the vast OJOG audience and alert the practising gynaecologist of such a rare but potentially easily treatable complication of Depo Provera use.
\end{abstract}

Keywords: Depo Provera; Cervical Stenosis; Heamatometra; Adolescence

\section{INTRODUCTION}

We present a case report managed in the secondary hospital setting of the United Kingdom. This case debates the use of Depo Provera in adolescence as a method of treating menorrhagia and debates the role of its role as a cause of cervical stenosis and haematometra.

\section{CASE REPORT}

A 17 year old virgo-intacta was referred by her general practitioner (GP), with a 3 year history of sudden flooding and dysmenorrhoea. She had been placed on depoprovera (medroxyprogesterone acetate) because of a history of menorrhagia. She had been amenorrhoeic on depo provera, prior to the history of sudden bouts of flooding. It had been noted in the past medical history that the patient suffered with migraine attacks complicated by aura.

Her GP had arranged a pelvic scan, which revealed a bulky axial and fluid filled uterus (fluid and solid material seen on scan) measuring $90 \times 47 \times 77 \mathrm{~mm}$ (Figure 1 attached as a separate file). The ovaries were noted to be polycystic in nature. A diagnosis of haematometra secondary to imperforate hymen was initially arrived at. The patient went on to having MRI scan which con-

\footnotetext{
"Corresponding author.
}

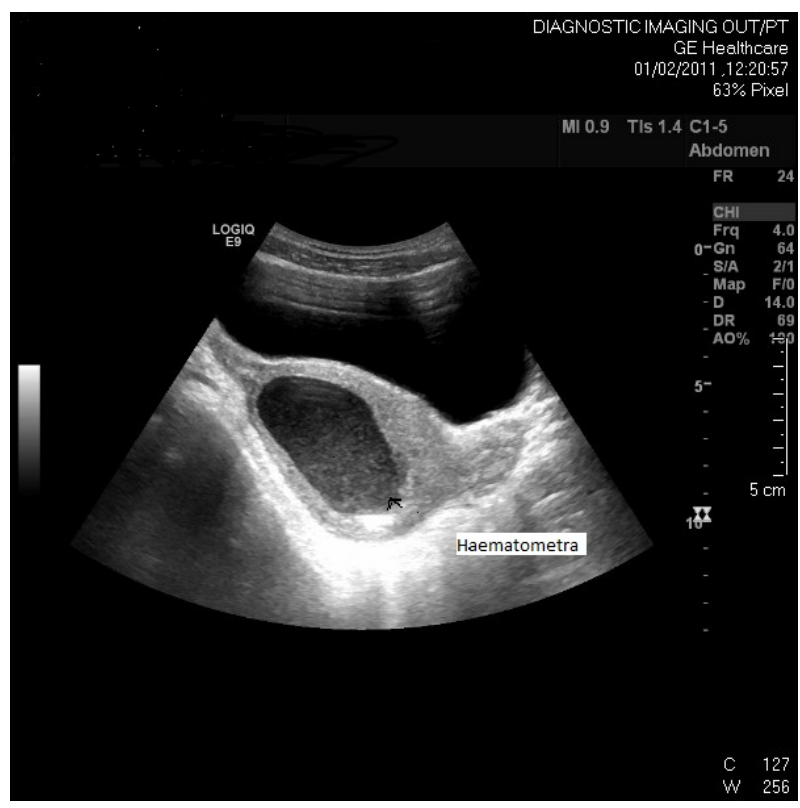

Figure 1. Diagnostic imaging: Haematometra.

firmed the above, but also that her renal tract appeared normal. She was admitted for an examination under anaesthesia, drainage of the uterine contents and insertion of a coil.

At surgery, she was found to have an intact hymen, which was not imperforate, the cervical os was stenotic and had to be dilated using lachrymal duct dilators initially, before using hager dilators, up to size 7 . Three hundred millilitres of old, altered blood was aspirated and a Nova T 380 coil was left in-situ and the threads cut to $2 \mathrm{~cm}$. She was continued on oral provera and seen in the clinic at 6 weeks to remove the coil. At the appointment she admitted that following initial heavy blood stained discharge, she had stopped bleeding, but just before the clinic she had experienced another bout of flooding. The threads of the coil were not seen on examination and a repeat scan confirmed that the uterus was once again fluid filled and the coil was seen within the cavity. A repeat EUA was performed, the cervical canal dilated again and another $250 \mathrm{mls}$ of altered and 
fresh blood was aspirated and the coil was removed.

She was scanned 6 weeks later and the uterine fluid dimensions were as follows: $50 \times 17 \times 17 \mathrm{~mm}$ and the endometrium was noted to be atrophic. The patient was then placed on Qlaira to aid re-growth of the endometrial lining, in the hope that this would prevent re-accumulation of blood, prevent re-stenosis of the cervical canal and flooding.

After being on Qlaira for 3 months, she was symptom free, having regular withdrawal bleeds and a scan confirmed that the uterus was of normal dimensions and had an endometrial thickness of $5 \mathrm{~mm}$.

\section{DISCUSSION}

Information about adolescent cervical stenosis is lacking as suggested by the Medline data base search. Inputting the search words "cervical stenosis", "adolescent cervical stenosis" and "depo provera” in the Medline data search engine "Pub Med" returned no relevant search results. Cervical stenosis most often occurs in the region of the internal os and it could be secondary to periodic injections of medroxyprogesterone (Depo Provera) [1].

The Society of Canadian Colposcopists suggests that Cervical stenosis occurs mainly in hypo estrogenic states such as Reproductive age group women with oligomenorrhea or amenorrhea, women on oral contraceptives with oligomenorrhea or amenorrhea, women who are postpartum and/or breast feeding as well as women on Depo Provera and postmenopausal women who are not on HRT [2].

Medroxyprogesterone acetate (Depo Provera ${ }^{\circledR}$ ) is a commonly used drug in gynaecology. Its contraceptive properties are well known making it a convenient parenteral method of contraception. It is a common misconception that the secondary amenorrhoea that the drug often induces makes it an appropriate medication to treat heavy periods. Right the opposite; menstrual disturbances including risk of heavy or prolonged bleeding can complicate a course of treatment. Undiagnosed vaginal bleeding is considered a contraindication [3].

According to the British National Formulary, in adolescents, medroxyprogesterone acetate (Depo Provera ${ }^{\circledR}$ ) should only be used when other methods of contraception are inappropriate, while according to the Faculty of Sexual and Reproductive Healthcare guideline on Depot Provera use In adolescents, Depo Provera may be used as a first-line contraception but only after other methods have been discussed with the patient and considered to be unsuitable or unacceptable. There is no mention in the guideline about the appropriateness of use of Depo Provera as a first line medication to treat menorrhagia [3,4].

The most recent NICE (National Institute of Clinical Excellence) guideline on treating heavy menstrual bleeding suggests that if pharmacological treatment in this patient should have been instituted initially with Tranexamic acid or non-steroidal anti-inflammatory drugs (NSAIDs) or combined oral contraceptives or as a second line treatment Norethisterone $(15 \mathrm{mg})$ daily from days 5 to 26 of the menstrual cycle, or injected longacting progestogens can be used. If hormonal treatments are not acceptable to the woman, and then either Tranexamic acid or NSAIDs can be used [5].

NICE's very first line of treatment for heavy periods - the Mirena IUS seemed inappropriate in this case (due to the virgo intacta state of the patient), one could question why alternatives such as Tranexamic Acid, NSAIDs or Norethisterone were not used as a first line of treatment [5].

It is therefore debatable whether Depo-Provera should have been started at all as a first line medication to treat menorrhagia in this case. Having frequent migraines' complicated with aura would have been a contraindication for oestrogen containing medication, such as the COC pill, as the drug falls into the UKMEC Category 4 - "Unacceptable health risk and should not be used" [6].

Therefore we feel further exploring the symptoms of aura complicated migraine should have been undertaken, in view of starting the COC pill should true aura seemed unlikely.

\section{CONCLUSION}

In hindsight it would appear that the symptoms of haematometra were exacerbated by the Depo Provera use, as stopping the medication rendered a quick resolution in the patient's symptoms. One could also question whether this patient had true migraine attacks complicated by aura, as the Qlaira ${ }^{\circledR}$ (Estradiol Valerate and Dienogest) did not seem to worsen the already existent migraine and arguably corrected the hypo estrogenic state of the patient thus reverting the symptoms of amenorrhoea and haematometra.

\section{REFERENCES}

[1] Katz, V. (2007) Comprehensive gynecology. 5th Edition, Mosby, Inc., Maryland Heights.

[2] Lickrish, G.M. and Bryson, P. (2005) How to prevent cervical stenosis after excision. SCC at the SOGC's 24th Ontario CME, Toronto, 24-26 November 2005.

[3] (2011) Electronic BNF No. 62, September 2011.

[4] Statement on MHRA guidance on depo-provera (2004) Faculty of Sexual \& Reproductive Healthcare, 18 November 2004.

[5] (2007) Heavy menstrual bleeding. NICE Guidline, London, January 2007.

[6] (2007) Combined oral contraception-First prescription guideline. Faculty of Sexual \& Reproductive Healthcare, January 2007. 